\title{
Intracranial Germinomas of Unusual Location. Combined Management: A Series of 3 Cases from the Central Military Hospital, Bogota Colombia
}

\author{
Harold Palmera Pineda ${ }^{1 *}$, Diana Paola Duarte ${ }^{1}$, María Camila Vargas ${ }^{2}$, Lina María Jiménez ${ }^{2}$, \\ Luis Orlando Rojas ${ }^{3}$ \\ ${ }^{1}$ Resident Neurosurgery, Military University of Nueva Granada, Central Military Hospital, Colombia \\ ${ }^{2}$ Medicine Student, Military University of Nueva Granada, Colombia \\ ${ }^{3}$ Neurosurgery Specialist Central Military Hospital, Colombia
}

*Corresponding Author: Harold Palmera Pineda, Resident Neurosurgery, Military University of Nueva Granada, Central Military Hospital, Colombia.

\begin{abstract}
Intracranial germinomas are rare in the general population, with the pediatric population being the age group where they occur most frequently. Clinical manifestations are usually diverse and nonspecific, diagnostic images are an important tool, however, when the location is unusual, the histological diagnosis remains the gold standard. Despite being malignant tumors, present an adequate response to treatment, with high survival rates observed when radiotherapy is used within the management scheme. We report a series of cases of three patients treated at the Central Military Hospital of Bogota Colombia.
\end{abstract}

Keywords: Germinomas, cerebral midline, embryonic phase, radiotherapy, Central Military Hospital

\section{INTRODUCTION}

Germinomas are acommon kind of germ cell tumor [1], the World Health Organization (WHO) classifies these lesions into six histological subtypes: germinoma, teratoma, embryonic carcinoma, choriocarcinoma, yolk sac tumor and mixed tumor [2]. Depending on the patient's age, this neoplasm makes up for approximately $0,1 \%$ to $3,4 \%$ of all intracranial tumors and $0,5 \%$ to $2,0 \%$ of all primary central nervous system (CNS) cancers in western countries and Asia, respectively [3].

Germionomas tend to appear in the midline structures of the brain, mainly the regions of the pineal gland, followed by structures such as the suprasellar cistern, basal ganglia and thalamus. During the embryonic phase, the main germ cells appear in the third week of pregnancy and they migrate dorsally starting from their point of origin in the yolk sac wall all the way to the gonadal outline. In this process of downward migration, some cells might reunite ectopically through the midline, in CNS, mediastinum, sacrococcygeal area, retroperitoneum and gonads. In either location, the germ cells can proliferate and experience a neoplastic transformation, giving place to a tumor which type will depend on the degree of differentiation that it may have experimented up until that point, with a different prognosis according to its clinical, histological and biological presentation [4]. Regardless of this prevailing location there are reports of lesions on the synchronous pineal and neurohypophyseal region, there have also been reports of other rarer locations such as the basal ganglia, the cerebellar vermis, the pituitary fossa, the ventricular system and the optic chiasma [5].

Histologically, pure germinomas are made up of big undifferentiated polygonal cells with abundant cytoplasm arranged in nests separated by bands of connective tissue [6]. Cystic degeneration and necrosis can be characteristic of germinoma. Necrosis can happen in the form of individual cells, small microscopic foci or, in most extreme cases, topographic areas [7]. Germionomas are generally associated to high levels of alpha-fetoprotein (AFP) and beta human chorionic gonadotropin (beta-hCG) in both the cerebrospinal fluid and the serum [8]. 
The most common symptom in patients with germ cell intracranial tumors is headache followed by nausea and emesis. It has been identified that approximately half of all patients have hydrocephalus at the time of the diagnosis. Other common symptoms include polyuria and / or polydipsia, diplopia, alterations of visual acuity or campimetric cuts, fatigue, weight loss, medro failure and early puberty. The symptoms vary significantly according to the type of tumor, for instance pineal tumors usually manifest with symptoms derived from hydrocephalus, while suprasellar tumors have symptoms related to endocrinopathies [9].

Neuroimaging takes a very important role amongst diagnostic aid; particularly nuclear magnetic resonance (NMR) becomes essential in the diagnosis of germ cell tumors. Selfdiffusion coefficient imaging is a NMR sequence recently developed, it has been used to evaluate neurological diseases including tumoral lesions. The differentiation between germinomas and other types of tumors is critical, because patients with germinomas can have a more favorable prognosis due mostly to complementary therapy. As far as tumoral markers, such as AFP and beta-Hcg, they are useful indiagnostic orientation, however, histological examination is necessary to establish a definitive diagnosis [1-4-7-9].

Intracranial germinomas are quite sensitive to radiation. Most contemporary literature agrees that treatment exclusively or mixed with radiotherapy shows favorable results with a survival to 5 years superior by $90 \%$ in patients treated solely with radiotherapy. In comparison with other primary malign tumors of the CNS, germinomas show a favorable evolution in most cases, in spite of this there are factors contributing to a bad prognosis such as being above 12 years old and thoracic location [10].

Below there will be a series of three clinical cases treated at Hospital Militar Central de Bogotá Colombia of germinomas in pediatric population with diverse locations and clinical manifestations.

\section{CASE Report}

\subsection{Clinical Case 1}

The patient is a 10-year-old male who didn't have medical history relevant to the case, he came in with a clinical picture that had been evolving for two months, characterized by holocraneal headache with oppressive characteristics, moderate intensity related to an alteration in eye movements and blurry vision, the picture sharpens a week prior to the consultation due to increased visual deficit and tremor of upper right limb. After physical examination he presents paresis of the III left nerve, visual acuity 20/20 on both eyes, without other pathological findings. A brain magnetic resonance is performed and it shows an intense heterogeneous enhancement of the left thalamic peduncle contrast with slight contra lateral extension through the tegument and reactive edema. It exerts a compressive effect on neighboring structures (Figure 1).

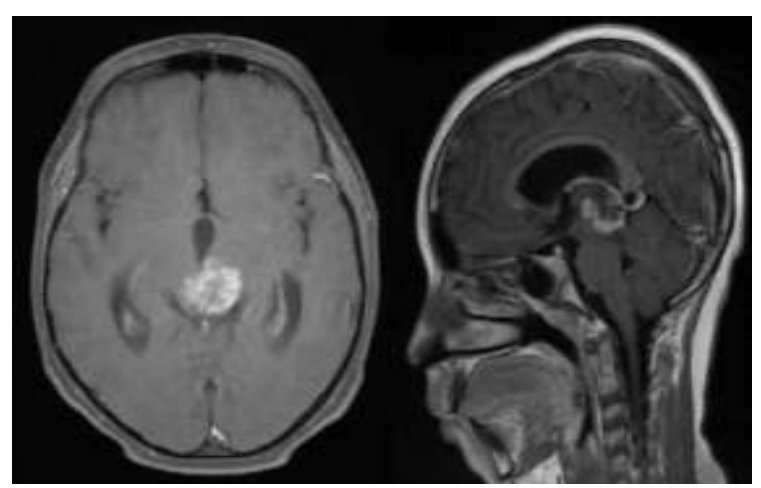

Figure 1

Due to imaging findings we decided to perform a stereotaxy guided biopsy that reported a germinoma negative for CD117 CAMP 5.2 HCG and AFP. Positive for PLAP. For that reason, treatment began with carboplatinetoposide induction chemotherapy (4 cycles) as well as 18 cycles of radiotherapy. During the follow up, the patient presented adequate evolution both clinically and in imaging, no evidence in imaging of control of residual or recurrent lesions. (Figure 2)

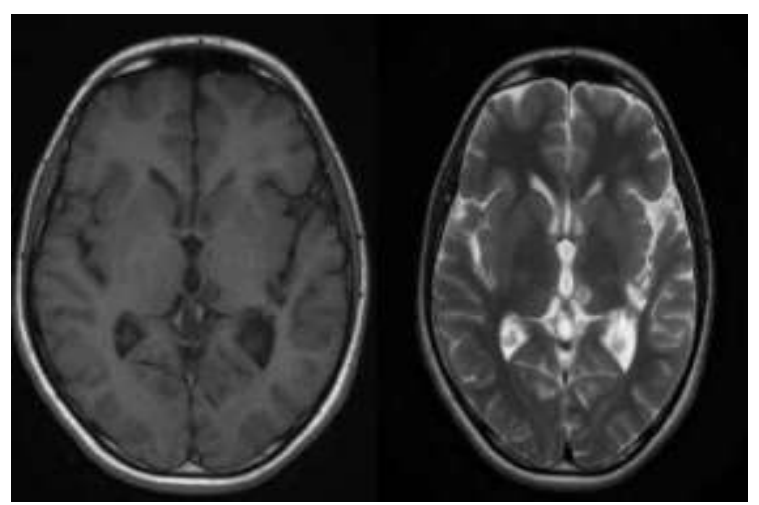

Figure 2

\subsection{Clinical Case 2}

The patient is an 11-year-old female with a clinical picture three months in evolution, 
characterized by stunted growth associated with behavioral alterations due to aggressiveness, apathy, social isolation and anorexia. Neurological physical examination showed no focal findings. NMR was performed, showing a large cystic lesion in the suprasellar region associated to incipient changes in supratentorial hydrocephalus (Figure 3).

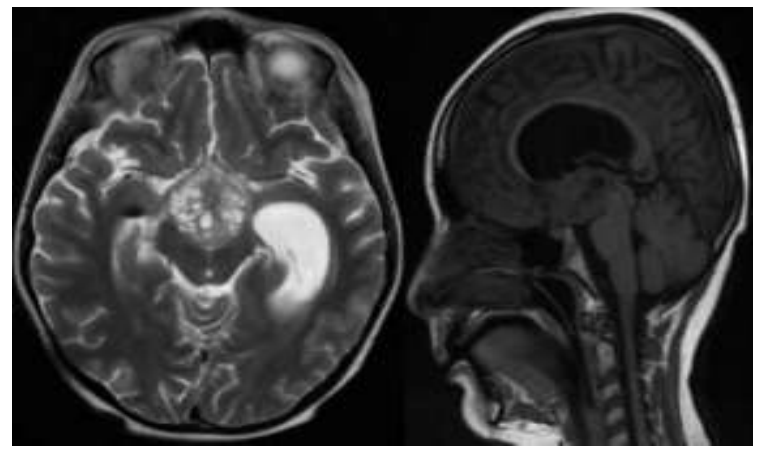

Figure 3

We decided to take a surgical approach through endoscopy for tumor resection and hydrocephalus treatment. In addition to this, she received complementary therapy with radiotherapy and chemotherapy that showed adequate response and favorable evolution, both clinically and in imaging (Figure 4).

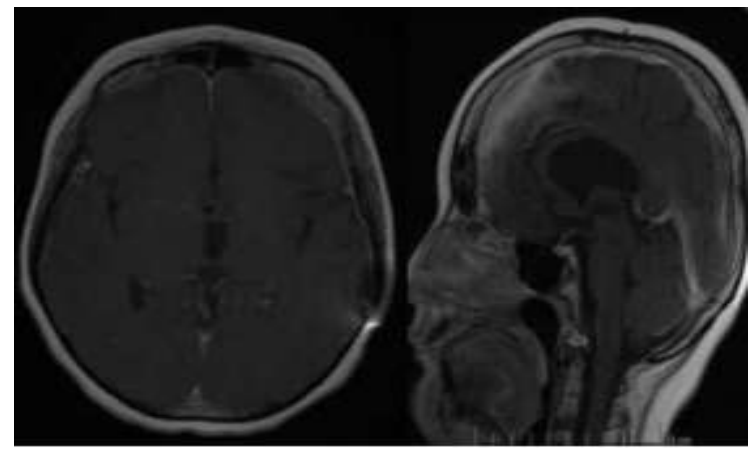

Figure 4

\subsection{Clinical Case 3}

The patient is a 12-year-old male without clinical history of relevance to the case, with a clinical picture that had been evolving for 10 months characterized by high intensity headache associated to abdominal pain and weight gain, eight months prior to consultation he presented polydipsia, polyphagia, polaquiuria and cold intolerance. After physical examination he presents generalized xeroderma without focal neurological deficit. Paraclinical testing indicated hyperprolactinemia, secondary hypothyroidism, and findings suggestive of diabetes insipidus. NMR is performed showing soft tissue formation in the suprasellar region in relation to pituitary stalk that enhances with contrast medium (Figure 5).

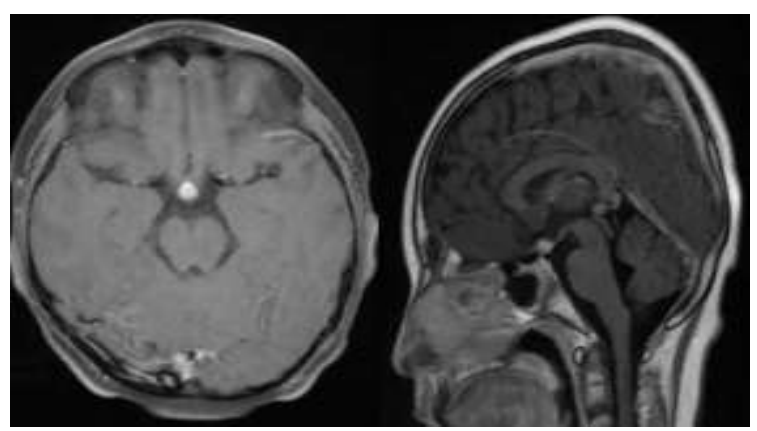

Figure 5

With these findings it is decided to take surgical treatment initially through endoscopy, however, due to technical difficulties during the procedure it cannot be completed and it is decided to take a second open surgical attempt through a pterional craniotomy, achieving a tumor resection and obtaining a pathology report that informs of a large cell neoplasm with nucleoli, another cell group made up of mature lymphoid cells, that suggest germinal neoplasia with CD117 focal positive markers, glial fibrillar protein negative, AFP negative, CK20 negative, CK7 negative. For that reason, treatment began with carboplatin-etoposide induction chemotherapy. During the follow up, the patient showed adequate evolution, both clinically and in imaging, with no evidence of tumoral residues or recurrences (Figure 6).

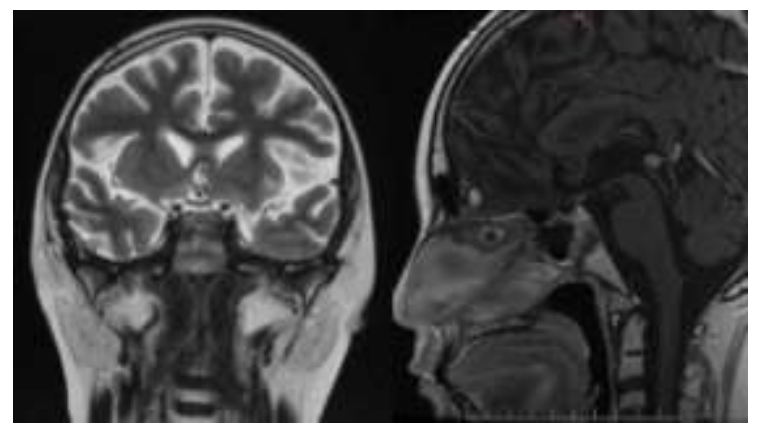

Figure 6

\section{DISCUSSION}

Intracranial germinomas are relatively rare entities, showing a higher incidence in pediatric population. The clinical presentation tends to be diverse, being mainly in relation with the location and the size of the tumor. The diagnosis tends to be difficult and, when its location is unusual, it needs histological confirmation. The focal point of treatment is usually radiotherapy that shows an adequate response and a favorable prognosis. In our case series it is clear that the 
clinic is usually completely unspecific, that when the location is unusual the diagnosis through imaging is not possible on its own. On top of that, it is also important to highlight that although radiotherapy is the primary treatment for intracranial germinomas, surgical management in its multiple modalities such as stereotactic biopsy, and open and endoscopic resections, can be an important tool for both the diagnosis as well as the treatment of these pathologies. Finally, these cases show how multidisciplinary work and the application of adequate and timely treatment generates a great impact in the patient's response and prognosis.

\section{REFERENCES}

[1] Zhengxiang Luo, Zhongrun Qian, Kun Yang, Hongyi Liu, Wenbin Zhang YZ. Primary Germinoma Originating from the Insular Lobe: A Case Report and Review of the Literature. World Neurosurg. 2017;98(February):871.e1871.e7.

[2] Samantha Shankar, Xiao Wu, Vivek B. Kalra, Anita J. Huttner AM. Ectopic intracranial germinoma. J Clin Neurosci. 2016;31:192-5.

[3] Horowitz MB HW. Central Nervous System Germinomas: A Review. Arch Neurol. 1991;48:652-657.
[4] Nuria Pardo Garcíaa AMV y M. SMR. Tumores de células germinales . Clin Transl Oncol. 2005;7(8):361-9

[5] Liang L, Korogi Y, Sugahara T et al. MRI of intracranial germ-cell tumours. Neuroradiology. 2002;44:382.

[6] Rosenblum MK, Nakazato Y MM. CNS germ cell tumors. In: WHO Classification of Tumours of the Central Nervous System. WHO Publ Center, Albany, NY. 2007;197.

[7] Douglas-Akinwande AC, Ying J, Momin Z et al. Diffusion-weighted imaging characteristics of primary central nervous system germinoma with histopathologic correlation: a retrospective study. Acad Radiol. 2009;16:1356.

[8] Ogino H, Shibamoto Y, Takanaka T et al. CNS germinoma with elevated serum human chorionic gonadotropin level: clinical characteristics and treatment outcome. Int $\mathbf{J}$ Radiat Oncol Biol Phys. 2005;62:803.

[9] Sethi RV, Marino R, Niemierko A et al. Delayed diagnosis in children with intracranial germ cell tumors. J Pediatr. 2013;163:1448.

[10] Lément Vialatte de Pémille, Franck Bielle, Karima Mokhtari, Esma Kerboua, Claire Alapetite AI. Basal Ganglia Germinoma in an Adult. World Neurosurg [Internet]. 2016;92:584.e11-584.e14. Available from: http://dx.doi.org/10.1016/j.wneu.2016.05.041

Citation: Harold Palmera Pineda, Diana Paola Duarte, María Camila Vargas, Lina María Jiménez, Luis Orlando Rojas. Intracranial Germinomas of Unusual Location. Combined Management:A Series of 3 Cases from the Central Military Hospital, Bogota Colombia. ARC Journal of Clinical Case Reports. 2020; 6(1):1316. doi:dx.doi.org/ 10.20431/2455-9806.0601004.

Copyright: (1) 2020 Authors. This is an open-access article distributed under the terms of the Creative Commons Attribution License, which permits unrestricted use, distribution, and reproduction in any medium, provided the original author and source are credited. 